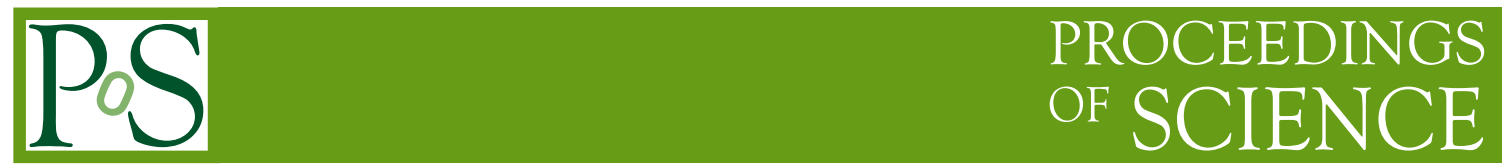

\title{
Studies of meson-like exotic states at LHCb
}

\section{Andrii Usachov* on behalf of the LHCb collaboration}

Laboratoire de l'accélérateur linéaire, Orsay / IN2P3 / CNRS and Université Paris-Sud

E-mail: andrii.usachovecern.ch

The LHCb experiment exploits decays of beauty hadrons as well as prompt production in protonproton collisions to investigate exotic mesons, especially in the charmonium mass region. Using large data samples collected during the Run I and Run II LHCb investigates exotic states to better understanding QCD. Recent results on exotic meson spectroscopy are presented.

XXXIX International Conference on High Energy Physics,

3-11 July 2018

Seoul, South Korea

${ }^{*}$ Speaker. 
The exotic mesons such as tetraquarks and hadronic molecules, which consist of four quarks are predicted by QCD. In order to study possible exotic mesons and cusp structures the $\mathrm{LHCb}$ experiment has performed a series of measurements by looking for intermediate resonances in the exclusive $B$ and $B_{s}^{0}$ mesons decays, as well as for hadroproduction of exotic hadrons. Searches for purely hadronic decay modes of the $X(3872)$, observations of exotic candidates decaying to $J / \psi \phi$, studies of the $Z(4430)^{-}$and search for the $X(5568)^{-}$, as discussed in this proceeding have been performed using a data sample collected at $\sqrt{s}=7$ and $8 \mathrm{TeV}$ with an integrated luminosity of $\int \mathscr{L} d t=3 \mathrm{fb}^{-1}$. The search for fully beautiful tetraquark $X_{b b \bar{b} \bar{b}}$ has been performed using a data sample collected at $\sqrt{s}=7,8$ and $13 \mathrm{TeV}$ with an integrated luminosity of $\int \mathscr{L} d t=6.3 \mathrm{fb}^{-1}$.

The $X(3872)$ state is the exotic hadron [1,2], which has been observed in different production processes, contrary to most of other exotic hadron candidates. The LHCb collaboration performed a series of measurements clarifying the nature of the $X(3872)$ : determined its quantum numbers to be $1^{++}[3,4]$, observed the $X(3872) \rightarrow \psi(2 S) \gamma$ decay [5], which indicates charmonium component in the $\mathrm{X}(3872)$, and measured its prompt production in pp collisions [6]. Recently, LHCb performed searches of $X(3872)$ decays to hadrons. The $X(3872) \rightarrow p \bar{p}$ decay has been searched with no signal observed [7]. The $X(3872) \rightarrow \phi \phi$ decay has been searched using a sample of inclusive b-hadron decays [8], yielding an upper limit at the $95 \%$ (90\%) confidence level of:

$$
\frac{\mathscr{B}(b \rightarrow X(3872) X) \times \mathscr{B}(X(3872) \rightarrow \phi \phi)}{\mathscr{B}\left(b \rightarrow \chi_{c 1} X\right) \times \mathscr{B}\left(\chi_{c 1} \rightarrow \phi \phi\right)}<0.39(0.34) .
$$

Studies of exotics candidates decaying to $J / \psi \phi$ at $\mathrm{LHCb}$ have been performed using a data sample of $B^{+} \rightarrow J / \psi \phi K^{+}$decays $^{1}[9,10]$. A full six-dimensional amplitude analysis has been performed. The fit assuming contributions only from all possible $K^{*+}$ states decaying to $\phi K^{+}$fails to describe the data yielding a p-value below $10^{-7}$. Contributions from the four states decaying to $J / \psi \phi$ are needed with significance of more than $5 \sigma$ to describe the data. The fit, which includes contributions from these states gave a p-value of 0.22 ; the result of the fit is shown on Fig. 1. The summary of the measured masses ${ }^{2}$, natural widths and established quantum numbers of observed exotics candidates are listed in Tab. 1. Further investigations with a larger data sample are needed to test cusp hypotheses of observed structures [11].

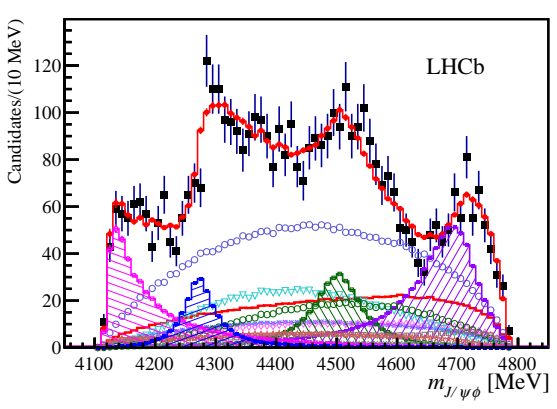

Figure 1: The projection of the fit (red line) to $M(J / \psi \phi)$ distribution in $B^{+} \rightarrow J / \psi \phi K^{+}$decays. Contributions from X(4140) (magenta), X(4274) (blue), X(4500) (green) and X(4700) (violet) are shown with filled hatches.

\begin{tabular}{c|c|c|c} 
& $M, \mathrm{MeV}$ & $\Gamma, \mathrm{MeV}$ & $J^{P C}$ \\
\hline$X(4140)$ & $4146.5 \pm 4.5_{-2.8}^{+4.6}$ & $83 \pm 21_{-14}^{+21}$ & $1^{++}$ \\
$X(4274)$ & $4273.3 \pm 8.3_{-3.6}^{+17.2}$ & $56 \pm 11_{-11}^{+8}$ & $1^{++}$ \\
$X(4500)$ & $4506 \pm 11_{-15}^{+12}$ & $92 \pm 21_{-20}^{+21}$ & $0^{++}$ \\
$X(4700)$ & $4704 \pm 10_{-24}^{+14}$ & $120 \pm 31_{-33}^{+42}$ & $0^{++}$
\end{tabular}

Table 1: Mass $(M)$, natural width $(\Gamma)$, quantum numbers $J^{P C}$ of observed exotics candidates decaying to $J / \psi \phi$.

\footnotetext{
${ }^{1}$ The inclusion of charge-conjugate states is implied throughout

${ }^{2}$ Natural units are used throughout the paper
} 
A four-dimensional amplitude analysis has been performed to study the $Z(4430)^{-}$state using a data sample of $B^{0} \rightarrow \psi(2 S) \pi^{-} K^{+}$decays [12]. The fit assuming contributions only from possible $K^{0 *}$ states decaying to $K^{+} \pi^{-}$failed to describe the data. Therefore, a contribution from $Z(4430)^{-} \rightarrow \psi(2 S) \pi^{-}$is needed, yielding a significance of the $Z(4430)^{-}$of at $13.9 \sigma$. The measured mass $M_{Z(4430)^{-}}=4475 \pm 7_{-25}^{+15} \mathrm{MeV}$ and width $\Gamma_{Z(4430)^{-}}=172 \pm 12_{-34}^{+37} \mathrm{MeV}$ are compatible with and more precise than the measurement by Belle [13]. The quantum numbers are established to be $J^{P}=1^{+}$with a significance more than $9 \sigma$ over the alternative hypothesis. More data are needed to distinguish between resonance and cusp hypotheses in future. In addition, the model independent confirmation of $Z(4430)^{-}$by moments analysis is performed [14].

The $Z(4430)^{-}$state may similarly be found in the $B_{s}^{0} \rightarrow \psi(2 S) \pi^{-} K^{+}$decays. The LHCb experiment has made an observation of $B_{s}^{0} \rightarrow \psi(2 S) \pi^{-} K^{+}$, and the relative branching fraction with respect to corresponding $B^{0}$ decay has been measured to be:

$$
\frac{\mathscr{B}\left(\bar{B}_{s}^{0} \rightarrow \psi(2 S) K^{+} \pi^{-}\right)}{\mathscr{B}\left(B^{0} \rightarrow \psi(2 S) K^{+} \pi^{-}\right)}=5.38 \pm 0.36(\text { stat }) \pm 0.22(\text { syst }) \pm 0.31\left(f_{s} / f_{d}\right) \%
$$

where the first uncertainty is statistical, second is systematical and the last uncertainty is related to the b-quark fragmentation ratio $f_{s} / f_{d}$. Similar measurement has been done in the $K^{*}(892)$ region for the $B_{s}^{0} \rightarrow \psi(2 S) K^{*}(892)$ decay mode. No signal for the $Z(4430)^{-}$has been observed in the $\psi(2 S) \pi^{-}$invariant mass distribution. The search of the $Z(4430)^{-}$in the $B_{s}^{0}$ decays will benefit from larger data sample collected at $13 \mathrm{TeV}$.

The $X(5568)^{-}$has been claimed by D0 collaboration decaying to $B_{s}^{0} \pi^{-}$[15]. The LHCb experiment has collected much larger data sample of $B_{s}^{0}$ mesons. No sign of $X(5568)^{-} \rightarrow B_{s}^{0} \pi^{-}$ has been observed for three different transverse momentum requirements at $\mathrm{LHCb}$ [16].

The fully beautiful tetraquark $X_{b b \bar{b} \bar{b}}$ below the $\eta_{b} \eta_{b}$ threshold has been searched in the decay $\Upsilon \mu^{+} \mu^{-}$using the $\Upsilon(1 S) \rightarrow \mu^{+} \mu^{-}$decay for normalisation [17]. No signal for the $X_{b b \bar{b} \bar{b}} \rightarrow$ $\Upsilon(1 S) \mu^{+} \mu^{-}$was observed. The fit to the $\Upsilon(1 S) \mu^{+} \mu^{-}$invariant mass is shown on Fig. 2. The upper limit has been extracted as function of assumed invariant mass of $X_{b b \bar{b} \bar{b}}$ as shown on Fig. 3.

In summary, the Run I data sample allows a series of measurements and searches for exotic hadrons with increasing sensitivity. A systematic study of the $X(3872)$ state are leading to understanding of its nature. Amplitude analysis of exclusive B-meson decays demonstrated that significant contributions from exotic candidates in the $J / \psi \phi$ and $\psi(2 S) \pi^{-}$spectra are needed. The properties of these states have been measured with better precision and the natural width of the

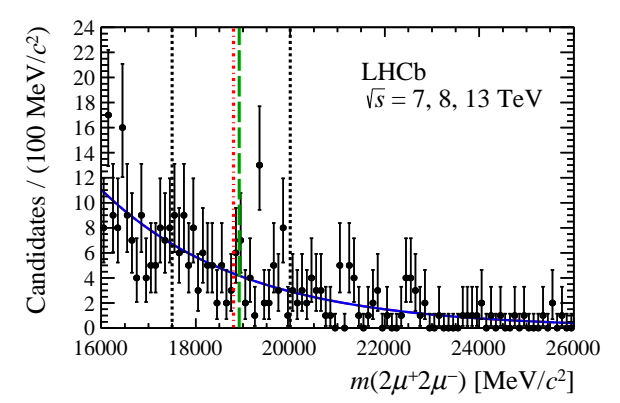

Figure 2: The fit to $M\left(\Upsilon(1 S) \mu^{+} \mu^{-}\right)$distribution. The blue line shows the curve of the fit, data points are shown in black. The red dashed line shows the $\eta_{b} \eta_{b}$ threshold, the green dashed line shown the $\Upsilon(1 S) \Upsilon(1 S)$ threshold.

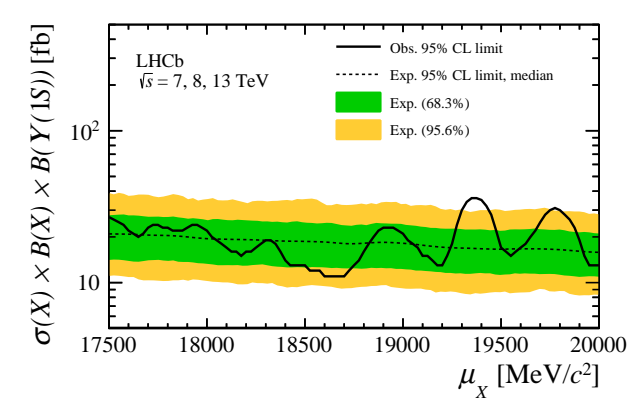

Figure 3: The 95\% upper limit (black line) for $X_{b b \bar{b} \bar{b}}$ production cross-section. The $\sigma(X)$ denotes the $X_{b b \bar{b} \bar{b}}$ production cross-section, the $B(X)$ denotes the branching fraction of the $X_{b b \bar{b} \bar{b}} \rightarrow \Upsilon(1 S) \mu^{+} \mu^{-}$decay, the $B(Y)$ denotes the branching fraction of the $\Upsilon(1 S) \rightarrow \mu^{+} \mu^{-}$decay. 
$X(4174)$ was measured to be much larger than the previous world average value. No sign of the $X(5568)^{-}$claimed by D0 has been observed by LHCb. The search of fully beautiful tetraquark has been performed using Run I and Run II data samples with no signal observed. The Run II data sample will allow to perform more measurements of $B_{s}^{0}$ decays and to improve precision of measurements presented here. Its higher statistics will be also used to search for other mechanisms of exotic hadron candidates production and improve understanding of their nature.

\section{References}

[1] N. A. Tornqvist, Isospin breaking of the narrow charmonium state of Belle at 3872-MeV as a deuson, Phys. Lett. B590 (2004) 209 [hep-ph $/ 0402237]$.

[2] C. Hanhart, Yu. S. Kalashnikova and A. V. Nefediev, Interplay of quark and meson degrees of freedom in a near-threshold resonance: multi-channel case, Eur. Phys. J. A47 (2011) 101 [1106.1185].

[3] LHCв collaboration, R. Aaij et al., Determination of the X(3872) meson quantum numbers, Phys. Rev. Lett. 110 (2013) 222001 [1302. 6269].

[4] LHCв collaboration, R. Aaij et al., Quantum numbers of the X(3872) state and orbital angular momentum in its $\rho^{0} J \psi$ decay, Phys. Rev. D92 (2015) 011102 [1504.06339].

[5] LHCB collaboration, R. Aaij et al., Evidence for the decay $X(3872) \rightarrow \psi(2 S) \gamma$, Nucl. Phys. B886 (2014) 665 [1404.0275].

[6] LHCB collaboration, R. Aaij et al., Observation of X(3872) production in pp collisions at $\sqrt{s}=7$ TeV, Eur. Phys. J. C72 (2012) 1972 [1112. 5310].

[7] LHCB collaboration, R. Aaij et al., Observation of $\eta_{c}(2 S) \rightarrow p \bar{p}$ and search for $X(3872) \rightarrow p \bar{p}$ decays, Phys. Lett. B769 (2017) 305 [1607.06446].

[8] LHCв collaboration, R. Aaij et al., Study of charmonium production in b-hadron decays and first evidence for the decay $B_{s}^{0} \rightarrow \phi \phi \phi$, Eur. Phys. J. C77 (2017) 609 [1706.07013].

[9] LHCB collaboration, R. Aaij et al., Amplitude analysis of $B^{+} \rightarrow J / \psi \phi K^{+}$decays, Phys. Rev. D95 (2017) 012002 [1606.07898].

[10] LHCB collaboration, R. Aaij et al., Observation of $J / \psi \phi$ structures consistent with exotic states from amplitude analysis of $B^{+} \rightarrow J / \psi \phi K^{+}$decays, Phys. Rev. Lett. 118 (2017) 022003 [1606 . 07895].

[11] E. Wang, J.-J. Xie, L.-S. Geng and E. Oset, Analysis of the $B^{+} \rightarrow J / \psi \phi K^{+}$data at low $J / \psi \phi$ invariant masses and the $X(4140)$ and $X(4160)$ resonances, Phys. Rev. D97 (2018) 014017 [1710.02061].

[12] LHCB collaboration, R. Aaij et al., Observation of the resonant character of the $Z(4430)^{-}$state, Phys. Rev. Lett. 112 (2014) 222002 [1404.1903].

[13] BELLE collaboration, K. Chilikin et al., Experimental constraints on the spin and parity of the Z(4430) ${ }^{+}$, Phys. Rev. D88 (2013) 074026 [1306.4894].

[14] LHCB collaboration, R. Aaij et al., Model-independent confirmation of the $Z(4430)^{-}$state, Phys. Rev. D92 (2015) 112009 [1510.01951].

[15] D0 collaboration, V. M. Abazov et al., Evidence for a $B_{s}^{0} \pi^{ \pm}$state, Phys. Rev. Lett. 117 (2016) 022003 [1602.07588].

[16] LHCB collaboration, R. Aaij et al., Search for structure in the $B_{s}^{0} \pi^{ \pm}$invariant Mass Spectrum, Phys. Rev. Lett. 117 (2016) 152003 [1608.00435].

[17] LHCB collaboration, R. Aaij et al., Search for beautiful tetraquarks in the $\Upsilon(1 S) \mu^{+} \mu^{-}$ invariant-mass spectrum, JHEP 10 (2018) 086 [1806.09707]. 